\title{
244th ENMC international workshop: Newborn screening in spinal muscular atrophy May 10-12, 2019, Hoofdorp, The Netherlands
}

\author{
Tamara Dangouloff ${ }^{\mathrm{a}}$, Arthur Burghes ${ }^{\mathrm{b}}$, Eduardo F. Tizzano ${ }^{\mathrm{c}, *}$, Laurent Servais ${ }^{\mathrm{a}, \mathrm{d}, * *}$, \\ on behalf of the NBS SMA Study Group ${ }^{1}$ \\ ${ }^{a}$ Division of Child Neurology, Centre de Références des Maladies Neuromusculaires, Department of Pediatrics, \\ University Hospital Liège \& University of Liège, 4000 Belgium \\ ${ }^{\mathrm{b}}$ Department of Biological Chemistry and Pharmacology, The Ohio State University, Columbus, OH, USA \\ ${ }^{\mathrm{c}}$ Department of Clinical and Molecular Genetics, University Hospital Valle Hebron, Medicine Genetics Group VHIR, \\ Pso. Vall d' Hebron, 119-129, 08035 Barcelona, Spain \\ ${ }^{\mathrm{d}}$ MDUK Neuromuscular Center, Paediatric Department, University of Oxford, Oxford OX1 2JD, UK
}

Available online $\mathrm{xxx}$

(C) 2019 Elsevier B.V. All rights reserved.

Keywords: Newborn screening; Spinal muscular atrophy; Pre-symptomatic.

\section{Introduction and overview}

A total of 19 participants including clinicians, newborn screening specialists, economists, geneticists, patients and patient advocates, and industry representatives from 12 countries convened from the May 12-14, 2019 in Hoofdorp, The Netherlands, for the 244th ENMC International Workshop, on the topic newborn screening (NBS) for spinal muscular atrophy.

Following a welcome from Alexandra Breukel, ENMC representative, and the chairpersons of the workshop, Eduardo Tizzano and Laurent Servais, Professor Tizzano gave an overview of the topic.

Spinal muscular atrophy (SMA) is an autosomal recessive disorder caused by abnormally low levels of functional survival of Motor Neuron (SMN) protein [1], which leads to

\footnotetext{
* Corresponding author.

** Correspondence to: CRMN Liège, CHR de la Citadelle, Boulevard du 12ème de Ligne, 4000 Liège, Belgium.

E-mail addresses: etizzano@vhebron.net (E.F. Tizzano), 1servais@chuliege.be (L. Servais).

${ }^{1}$ NBS SMA Study Group are listed at the end of the Article.
}

early death of motor neurons. SMN is coded by SMN1 gene, and $95 \%$ of patients present with homozygous absence of exon 7 of SMN1, whereas $5 \%$ present with the absence in one allele and a point mutation in the other allele [2]. Patients with SMA fall on a continuous spectrum of weakness: At one end are patients who present as neonates with rapid decrease in muscle strength that progresses to paralysis and to respiratory insufficiency; at the other are patients with adult onset SMA who have minor disabilities. The most severe form is called SMA type 0; it starts in utero, and patients are symptomatic at birth. SMA type 1 typically starts before the age of 6 months, and patients never acquire the ability to sit. SMA type 2 symptoms begin between 6 and 18 months, and these patients never gain the ability to walk. SMA type 3 starts after 18 months; patients walk initially but ambulation is lost in a high proportion of patients. In addition to this basic classification according to age and maximal ability achieved, groups may be further divided into many more subgroups, from 1.1 to $1.9,2.1$ to 2.9 ... adding more granularity in the classification and underlying the broad uninterrupted phenotypic spectrum from type 0 to type 4 [3]. All SMA types are associated with significant motor disability, a burden for caregivers, and substantial costs $[4,5]$. The main predictive factor for severity 
is the number of copies of $S M N 2$, a paralogous gene, from which a small amount of functional SMN protein is produced [6].

Recently, several therapeutic agents for treatment of SMA have been approved for clinical use or are in late-stage development. Spinraza (Nusinersen), an antisense oligonucleotide alters splicing of the $S M N 2$ pre-mRNA to cause expression of the full-length SMN protein. Spinraza was approved by the Food and Drug Administration (FDA) in December 2016 and by the European Medicines Agency in June 2017 as the first tailored therapy for SMA [7]. The benefit of therapeutic intervention soon after onset of symptoms has been demonstrated in double-blind, placebocontrolled study as well as during the real-world data collection [8-10]. Anecdotal cases of success in treatment of pre-symptomatic patients have been presented or published [11], and it has been reported that outcomes for 25 presymptomatic patients treated during an open-label study were dramatically more positive than for patients treated post-symptomatically [12]. Other approaches, including gene therapy and oral splicing modifiers currently in the late phase of clinical trial development [13-15], have also shown promising results [16]. The gene replacement therapy, known as Zolgensma, was approved by the FDA in May 2019 for treatment of patients with SMA below the age of 2 years [17], The benefit of early treatment with these agents in patients with SMA type 1 has also been suggested during ongoing studies.

Results from clinical trials indicate that early diagnosis should be facilitated. NBS for SMA meets the modified criteria proposed by Wilson and Jungner [18,19] which are widely used to determine whether screening for a disease should be included in an NBS panel. These criteria include 10 different items:

1. The condition sought should be an important health problem.

2. There should be an accepted treatment for patients with recognized disease.

3. Facilities for diagnosis and treatment should be available.

4. There should be a recognizable latent or early symptomatic stage.

5. There should be a suitable test or examination.

6 . The test should be acceptable to the population.

7. The natural history of the condition, including development from latent to declared disease, should be adequately understood.

8. There should be an agreed policy on whom to treat as patients.

9. The cost of case-finding (including diagnosis and treatment of patients diagnosed) should be economically balanced in relation to possible expenditure on medical care as a whole.

10. Case-finding should be a continuing process and not a "once and for all" project.
The recent availability of new treatments for SMA has provided impetuous to include screening for SMA in NBS programs [20-22]. Indeed, in the USA a number of states have implemented NBS and others are rapidly implementing it. (https://www.babysfirsttest.org/ newborn-screening/rusp-conditions\#spinal-muscular-atrophy) The aim of this workshop was to synthetize the currently available information on technical, ethical, economic, and practical aspects of NBS and to identify gaps in our current knowledge of patient identification and communication of diagnosis and treatment following NBS.

\section{Biomarkers and predictors}

Arthur Burghes gave an overview of the current SMA predictors. The first key modifier of the SMA phenotype is $S M N 2$ copy number. The second is the type of SMN2 present; specific variants such as c. $859 \mathrm{G}>\mathrm{C}$ in exon 7 and the intron 6 variant $\mathrm{A}-44 \mathrm{G}$ both alter the amount of fulllength SMN produced from SMN2 [23-26]. In general, there is strong correlation between the number of $S M N 2$ copies and SMA phenotype [6]. However, there are exceptions and only some of these are explained by variants in $S M N 2$ $[25,26]$. Other modifiers of SMA have been suggested, but there are no validated genetic markers yet that can be readily used in follow-up analysis to obtain a more accurate genotype versus phenotype correlation. In addition, it has been reported by several experts that the precise quantification of SMN2 number varies from one lab to another, especially for the highest number of copies. Indeed Schorling et al. [27] reported that on re-testing SMA patient samples, 9 out of 20 samples had a discrepant copy number determination. In experience of other expert labs, the observed discrepant copy number on retesting are usually consistent with phenotype. Issues concerning DNA quality and methodologies may underlie these discrepancies [6,27]. One particular concern is the rare occurrence of type 1 SMA cases with 4 or more copies of SMN2. Samples from these patients should be further analyzed to determine whether these diagnoses result from inaccurate copy number tests. In large collections of patients in laboratories with experience in $S M N 2$ determination, 4 copies of $S M N 2$ in type 1 has not been seen so it must be rare finding albeit its exact frequency is not known [6]. It would seem important to re-test these discordant samples to determine whether any patient with high $S M N 2$ copy number have severe illness as this is critical in risk assessment. It can be assumed that historical SMN2 copy number data and phenotype gives a reasonable indication that the genotype versus phenotype correlation can be used to calculate practical risk estimates [6].

Information on natural history and clinical evolution is crucial to facilitate decisions on benefits of early treatment. The main question after a newborn screen that is positive for $S M N 1$ deletion and a given copy number of $S M N 2$ is the probability of developing a particular form of SMA or of being asymptomatic for a long period of time [19]. The 
calculations for this risk use a multinomial logistic regression model. In this model both the SMN2 copy number and the type of SMA can be included and additional modifiers or factors such as SMN2 variants (i.e., c.859G $>$ C and A-44G) and gender can be included. Depending on the nature of the studies and the specifics of the dataset, a Bayesian calculation to determine posterior probability may be required, [6,28] which gives a more accurate probability of developing a specific phenotype. The known SMN2 variants account for some cases where phenotype is milder than would be expected based strictly on $S M N 2$ copy number [6,25,26]; however, there are a large number of cases that are milder than expected without these variants, and these are under active investigation to identify additional modifiers at the DNA level. It is likely that a number of these modifiers lie outside the SMN gene region. The identification of additional modifiers will improve prediction of phenotype by genotype and can be incorporated in the model.

Wildon Farwell discussed recent findings regarding neurofilaments in the context of NBS. Neurofilaments are intermediate filaments uniquely expressed in neuronal cells that, together with microtubules and microfilaments, make up the neuronal cytoskeleton [29,30]. Neurofilaments are composed of three proteins differentiated by molecular weight - light, medium, and heavy - that are released into the extracellular fluid during axonal degeneration [29,30]. Neurofilaments heavy and neurofilaments light have been proposed as biomarkers of disorders characterized by axonal injury and degeneration, and their clinical utility in diseases such as multiple sclerosis and amyotrophic lateral sclerosis is being explored [30]. Axonal neurofilaments are predominately phosphorylated, conferring resistance to protein degradation [29].

The natural disease course of SMA can now be significantly altered with treatment [7,31,32], and therefore strategies to assess disease activity and monitor treatment response are needed. Biogen has investigated plasma levels of phosphorylated neurofilaments heavy ( $\mathrm{pNF}-\mathrm{H})$ in children with and without SMA using blood samples collected at baseline from participants in Spinraza clinical trials (with presymptomatic, infantile-onset or later-onset SMA [ $n=302]$ ) and from children without SMA $(n=34)$, as well as change in pNF-H with treatment. The levels of pNF-H were measured using the ProteinSimple ${ }^{\circledR}$ enzyme-linked lectin assay [33]. In children without SMA, pNF-H levels were highest in the youngest infants and appeared to decline with age: median (range) in those aged $<1$ year was $1510 \mathrm{pg} / \mathrm{mL}$ (5797030; $n=6$ ) compared with $124.5 \mathrm{pg} / \mathrm{mL}$ (below the limit of quantification-395; $n=28$ ) in those aged 1 to 18 years $(p=0.0002)$ [34]. In children with SMA, baseline plasma pNF-H levels were higher than levels in children without SMA, and were highest in the youngest affected individuals; these individuals were pre-symptomatic and generally had two SMN2 copies [35]. In individuals with pre-symptomatic or infantile-onset SMA who received Spinraza treatment, pNF$\mathrm{H}$ levels declined during the loading period of Spinraza and then remained relatively stable over the course of the study [34,35]. In contrast, participants who received sham treatment experienced a steady, gradual decline in $\mathrm{pNF}-\mathrm{H}$ over the course of the study [34]. Results from additional analyses demonstrated the potential of pNF-H levels after initiation of treatment with Spinraza to predict future motor function improvement. Taken together, the results suggest that pNF-H may be a useful biomarker for predicting motor function outcomes in SMA. The utility of additional markers in cerebrospinal fluid of treated SMA patients is also matter of active research [36,37].

\section{Techniques of NBS}

François Boemer presented the validation of an inhouse technique for NBS designed to specifically recognize homozygous deletions of exon 7 in the SMN1 gene [22,38]. To ensure the specificity for SMN1 and to avoid any SMN2 gene detection, qPCR screening assay was designed with a specific locked nucleic acid probe. The newborn screening center in Liege, Belgium, initiated a 3-year pilot study to screen all newborns in its area on March 5, 2018. Thereafter, several improvements allowed shortening of the turnaround time. The technical refinements, the acquisition a new qPCR instrument, the hiring of a dedicated lab technician, and the extension of the project to all Southern Belgium allowed the delay between birth and results availability from 14.2 days when screening was initiated to the current 8.5 days.

Mikael Hjort presented PerkinElmer's objectives for NBS. Panel expansion to include screening for additional diseases, like SMA, and introduction of new technologies will present challenges to NBS laboratories. Use of liquid handlers, automation, and dedicated software solutions will become routine in NBS workflows.

SMA NBS assays detect SMN1 exon 7 deletion in a qualitative manner and carrier detection should be avoided. To ensure specificity of the SMA assay, modified nucleotides such as locked nucleic acid probes can be used to ensure specificity for SMN1. SMN2 copy number determination can be part of the NBS workflow as a second-tier testing utilizing droplet digital Polymerase Chain Reaction (PCR) technology; however, this should not be considered as a critical part of the NBS program as it can be also done outside the NBS laboratory during the confirmation stage.

The SMA NBS assay can be multiplexed together with the severe combined immunodeficiency screening assay. Furthermore, multiplexing of T-cell receptor excision circles, kappa-deleting recombination excision circles, and SMN1 can be done in one assay utilizing real-time PCR technology without increasing daily hands-on workload and complexity. The combination of simple DNA extraction, multiplexing, and automation allows maximum efficiency in the workflow.

During discussion, it was noted that none of the methods currently employed in NBS for SMA allows detection of point mutations in SMN1. Thus, about 5\% of cases will remain undiagnosed until manifestations of the disease occur and specific genetic tests are performed. 
Table 1

Ongoing SMA screening projects in Europe.

\begin{tabular}{|c|c|c|c|c|}
\hline & Germany & Italy & Belgium & Spain \\
\hline Type of consent & $\begin{array}{l}\text { Information of the parents, } \\
\text { additional Informed } \\
\text { consent }\end{array}$ & $\begin{array}{l}\text { Consent (information } \\
\text { during pregnancy, flyer, } \\
\text { social media camping, } \\
\text { consent signature) }\end{array}$ & $\begin{array}{l}\text { No consent (as for other } \\
\text { NBS), informed consent for } \\
\text { confirming }\end{array}$ & $\begin{array}{l}\text { Proposal of Information flyer, } \\
\text { informed consent for } \\
\text { confirming positive cases }\end{array}$ \\
\hline Type of assay & Real-time PCR & $\begin{array}{l}\text { Real-time PCR (dedicated } \\
\text { DBS) }\end{array}$ & $\begin{array}{l}\text { Real time PCR }+ \text { MLPA for } \\
\text { positive cases }\end{array}$ & $\begin{array}{l}\text { Real-time PCR coupled with } \\
\text { severe combined } \\
\text { immunodeficiency +MLPA } \\
\text { for positive cases }\end{array}$ \\
\hline Budget per infant screened & Non-available & 2.35-3.35 euros & 2.5 euros/sample + tech & \\
\hline Starting date & $15 / 01 / 2018$ & $15 / 03 / 2019$ & 05/03/2018 & Not yet \\
\hline $\begin{array}{l}\text { Expected births per year (pilot } \\
\text { duration) }\end{array}$ & $150,000 /$ year ( 3 years) & $70-80,000 /$ years $(2$ years $)$ & $60,000 /$ year ( 3 years) & $60-70,000 /$ year \\
\hline $\begin{array}{l}\text { Number of patients screened so } \\
\text { far }\end{array}$ & 178,000 & $>7000$ & 42,000 & 0 \\
\hline
\end{tabular}

\section{NBS programs}

Programs from the USA and several European countries were discussed, with the aims of comparing costs, organization, attitude towards informed consent, and techniques (Table 1).

Arthur Burghes gave a general overview of the status of SMA NBS in the USA. A number of states have started NBS for SMA, for instance Minnesota, Indiana, Utah and other states have legislation mandating this screening and some states such as Ohio are implementing it in pilot studies. A number of these states are using the real-time PCR assay developed by the Centers for Disease and Control to detect the loss of SMN1 exon 7. This assay was developed to be used in combination with the assay for detection of severe combined immunodeficiency (the TREC assay), which is also a DNAbased test. This allows rapid and inexpensive implementation of the assay as all states are performing severe combined immunodeficiency screening. The assay described above that was developed by PerkinElmer is very similar but the exact differences between the two cannot be determined due to lack of information concerning the primers and probes in the PerkinElmer assay. In the case of New York [21], the lack of $S M N 1$ is first determined and then the copy number of $S M N 2$ is determined concurrently with confirmatory tests. The aim is rapid reporting of the results to the physician to allow rapid treatment. To date, there are no data available on the number of babies screened or the number of patients identified.

Francesco Danilo Tiziano described the status of the Italian NBS for SMA. The pilot project that is ongoing in Italy was designed as an epidemiologic study with the goal of establishing the incidence of SMA in two regions: Lazio and Tuscany. Informed consent is required. Due to the duration of the study (two years) and the limited estimated number of patients (about 20), no formal analysis of treatment efficacy is planned. The pilot project is aligned to the extended metabolic
NBS that is mandatory by law, and exploits the same infrastructure for the management of samples. In contrast with the US and Belgian organization, a dedicated dried blood spot (DBS) is used for SMA. The diagnostic testing is centralized in a single center, the Catholic University in Roma. The genetic test is performed using an in-house assay based on real time PCR. The assay has been validated for carrier testing and SMN2 copy number assessment, has been adapted for an automatic analytic pipeline in small reaction volumes, and coamplifies SMN1 and SMN2 exon 7, which are differentiated by different Taqman MGB probes. $S M N 2$ is amplified as a PCR positive control. Positive samples are confirmed by a second DNA extraction from the same DBS. Subsequently, the family is invited for genetic counselling to explain the condition, prognosis, and therapeutic opportunities. On that occasion, a fresh blood sample is collected from the child, and an appointment is made for the neonate at a tertiary neuromuscular center, which will be kept assuming that confirmatory testing is positive. An official report, which includes the SMN2 copy number, is provided to the family in order to obtain the reimbursement of the treatment by the National Health System. The pilot project started in the single center for the exploratory phase on March 15, 2019: So far, over 7000 newborns have been screened, and no patients have been reported. The screening was extended to all newborn centers in July.

François Boemer described the implementation of the Belgian NBS. A 3-year pilot study was initiated on March 5, 2018 in a Belgian neonatal screening laboratory to cover 17,000 newborns per year [22]. The extension of the program's coverage to the whole of southern Belgium (French-speaking Belgium: Wallonia-Brussels federation), extending the population's coverage to 55,000 babies per year, was carried out 9 months after the launch of the pilot study. The SMA NBS follows the general NBS process, using the same DBS. Similarly, to any screening in Southern Belgium, 
parents are informed of the existence of a screening for several diseases and have the option to opt out; parents rarely choose not to have the screening. Formal signed consent is not required. After more than one year of screening, 42,000 newborns have been tested for SMA. Six cases of SMA were identified: two children with two copies of $S M N 2$, two children with three copies, and two with four copies. This corresponds to an incidence of 1 in 7000. All identified patients have received treatment with Spinraza or have been included in a gene or oral therapy trial and are being monitored by clinical and physical therapy tests including the CHOP-intend and the Bayley test (motor and cognition). A medical-economic analysis of the project is being carried out, with questionnaires on quality of life and cost caused by the disease. Over the course of the project, public engagement through mainstream media and Facebook (http://facebook/sunmayariseonsma) are ensuring visibility.

Wolfgang Müller-Felber reported on the German pilot project based on PCR of DBS samples [39]. Starting in January 2018 the project, which is still going on in three centers in the southern and western part of Germany (Munich, Essen, and Münster) and which was started as a collaboration project with the Cystinosis Foundation, had screened 178,000 newborns through by the end of March 2019. Twenty-five children with homozygous mutations in the SMN1 gene were identified for an incidence of 1 in 7120. Sixteen children (64\%) had two or three copies of SMN2, and the remaining four and more copies. Treatment was offered to all children with two or three $S M N 2$ copies. In 12 children treatment with Spinraza was started immediately after confirmation of the diagnosis. All children are undergoing regular clinical and physiotherapeutic tests. HINE and CHOP-Intend scores show near normal development in all children who began treatment prior to onset of symptoms. In one child with four copies of SMN2 symptoms appeared at 10 months of age. NBS was well accepted by the parents. The project will be continued until NBS is part of the general NBS program in Germany.

Eduardo Tizzano presented a proposal for a NBS project in Catalonia. Among the different programs of NBS in Spain there is a large heterogeneity in the 17 autonomous regions that are responsible for new program implementation, with screening for 7 to up to 30 diseases depending on the region. Catalonia has approximately 7,500,000 inhabitants and between 60,000 and 70,000 births per year. NBS screening in Catalonia started in 1968 and includes 24 diseases. Catalonia was the first region in Europe to include severe combined immunodeficiency by T-cell receptor excision circles determination, to which the SMA NBS could be coupled. Initial guidance to run a pilot project is under review by health authorities responsible for the regional NBS program. The proposal is to initially conduct SMA screening through the overall process of NBS, and the analysis will be centrally performed. A flyer about the purpose of this pilot study will be included in the initial information that is given to the families during the collection of blood, and families may choose not to participate. Informed consent will be collected at the stage of diagnosis confirmation, which will be performed in the reference center. Communication of diagnosis is considered to be a crucial issue in the project and is expected to be provided by an expert team of genetic counselors and pediatric neurologists with psychological support for decision making.

Kacper Rucinski described the ongoing efforts to initiate pilot projects of SMA NBS in Central and Eastern Europe. Pilot projects in Hungary and Poland were outlined. The Polish project aims to screen 120,000 newborns over two years and is in a more advanced stage of planning than is the Hungarian program.

\section{Treatments}

Enrico Bertini presented the data from the Biogensponsored NURTURE study (NCT02386553) of Spinraza efficacy. Spinraza was the first approved treatment for SMA. Interim results were presented as of May 2018. Treatment was initiated prior to symptom onset in infants with two or three $S M N 2$ copies. Enrolled infants were aged $\leq 6$ weeks at first dose, clinically pre-symptomatic, had a compound muscle action potential (CMAP) above $1 \mathrm{mV}$ and were genetically diagnosed with SMA. The primary endpoint is time to death or respiratory intervention ( $\geq 6 \mathrm{~h} /$ day continuously for $\geq 7$ days or tracheostomy). A total of 25 infants (2 copies $S M N 2$, $n=15$; 3 copies, $n=10$ ) were enrolled with a median age at last visit of 26.0 months (range 14.0-34.3 months). As of May 2018, all infants were alive, and none required permanent ventilation. Four infants (all with $2 S M N 2$ copies) required respiratory intervention for $\geq 6 \mathrm{~h} /$ day continuously for $\geq 7$ days during acute, reversible illness. All infants achieved the WHO motor milestone sitting without support, 22 of 25 (88\%) achieved walking with assistance, and 17 of $22(77 \%)$ were walking alone [12]. pNF-H levels rapidly declined during the Spinraza loading phase and then stabilized. At baseline, pNF$\mathrm{H}$ were considerably elevated in individuals with two copies of SMN2. Adverse events occurred in all infants; 20 of 25 had adverse events that were mild or moderate in severity; nine had severe adverse events. No new safety concerns were identified. There was continued benefit to infants who initiated Spinraza before symptom onset, emphasizing the value of early treatment made possible by NBS. This open ongoing trial will also identify biomarkers to use in the presymptomatic treatment approach.

Imran Kausar presented data on the AveXis AAV9-based gene therapy Zolgensma (previously known as AVXS-101) pre-symptomatic clinical trial (SPR1NT, NCT03505099) in SMA. The data are the most recent data cut from the ongoing SPR1NT trial and were recently presented at the American Association of Neurology meeting in Philadelphia, PA, USA. SPR1NT is a Phase 3, open-label, single-arm, multi-center trial designed to evaluate the safety and efficacy of a onetime intravenous infusion of Zolgensma in pre-symptomatic patients with SMA and two or three copies of SMN2 who began treatment at $\leq 6$ weeks of age. The primary outcome measure for patients with two copies of SMN2 is independent sitting for $\geq 30 \mathrm{~s}$ by 18 months. The primary outcome measure 
for patients with three copies of $S M N 2$ is standing without support for at least three seconds by 24 months.

As of March 8, 2019, all patients (18/18) were alive and event-free. One patient was enrolled into SPR1NT with four copies of SMN2 and was assessed for safety but not efficacy as this patient did not meet the intent-to-treat criteria. The median duration of follow-up was 5.4 months, and the median age was 6.1 months. Among patients with two copies of SMN2 $(n=8)$, a mean 8.9-point improvement from baseline in CHOP-INTEND was achieved 1 month post dosing, and a mean score of 8.4 points in Bayley-III Gross Motor was achieved by 2 months after dosing. All patients achieved or maintained a CHOP-INTEND score of 50 points, with four patients achieving a score of 60 points and three patients achieving the maximum score of 64 .

Patients with two copies of SMN2 reached age-appropriate motor milestones, including four patients who could sit without support for at least $30 \mathrm{~s}$ according to Bayley-III Gross Motor criteria, and one patient who could stand with assistance for $\geq 2 \mathrm{~s}$. Untreated natural history indicates that most patients with two copies of SMN2 never sit without assistance.

Serious adverse events were cases of croup $(n=1)$, lethargy $(n=1)$, and hypercalcemia $(n=1)$, all of which resolved and were considered unrelated to treatment by investigators. Other observed adverse events included elevated transaminases, elevated blood creatine phosphokinasemuscle/brain, and elevated troponin.

Ksenija Gorni presented the study design and the inclusion/exclusion criteria of the RAINBOWFISH trial (NCT03779334) designed to evaluate efficacy, safety, pharmacokinetics, and pharmacodynamics of Risdiplam. Risdiplam is a small-molecule splicing modulator that will be given orally. Infants with one to four copies of $S M N 2$ will be included in the study, and the primary analysis will be performed considering at least 10 babies with two SMN2 copies. Similar, to other studies in pre-symptomatic infants with SMA, the inclusion age will be $\leq 6$ weeks at time of first dose. Enrollment into this study has just begun and no data are available.

The use of NBS modalities has been discussed for RAINBOWFISH, considering the global nature of the study, which involves geographies with differing standard of care. NBS that is being utilized will follow an ad hoc approach using existing NBS programs when present and supporting ongoing pilots and starting new efforts where NBS is not readily available.

\section{Alternatives to NBS}

Eduardo Tizzano presented the carrier screening concept as an alternative to NBS. NBS is considered a reasonable initial approach to prevent and modify the trajectory of SMA, given the movement from treating patients with manifesting disease, which constitutes a tertiary prevention in patients with early symptoms, to a secondary prevention in asymptomatic patients. However, it is envisaged that in the future there will be consolidation of carrier screening programs in the population for primary prevention of SMA [19]. The final result will be a decrease of the incidence and prevalence of the disease with a potential positive impact on the health system considering the burden of the disease and the high cost of new treatments [40]. Carrier screening in SMA may be triggered by a family history of SMA or as part of a general screening programs. These programs could be SMA specific, as are the geography- or population-based screenings conducted for carriers of recessive conditions that are observed in limited locations or ethnic groups (e.g., Tay Sachs [41], cystic fibrosis [42,43], thalassemia [44]), or as part of expanded (commercially available) carrier screening next generation sequencing genetic panels that are offered for several autosomal recessive conditions. This type of screening is done in potential gamete donors in some fertility and reproduction clinics [45].

Parents of SMA patients are not always carriers since a small proportion of patients have pathogenic variants that occurred de novo or that resulted from germinal/somatic mosaicism [46]. Thus, even a universal carrier screening program will not make SMA disappear. SMA carrier detection should be a quantitative method that detects one copy of $S M N 1$ in classical 1/0 carriers. A small proportion of carriers have two SMN1 copies in cis and none on the other allele ( $2 / 0$ carriers). The current carrier diagnosis methods based on the SMN1 dosage do not allow discrimination between 1/1 non-carriers and 2/0 carriers when an individual shows two SMN1 copies. Although the presence of some polymorphisms in SMN1 can aid in categorization of a risk group of $2 / 0$ carriers [47], its absence in a person with two SMN1 copies does not preclude $2 / 0$ carrier status limiting the utility of this analysis to some populations [48]. Testing of parents to exclude the presence of a chromosome with more than one SMN1 copy is usually confirmatory to interpret the case under study as $1 / 1$ non-carrier [46,48].

There was an overall agreement that NBS and carrier screening programs are two complementary approaches to SMA prevention. Carrier screening by next generation sequencing has the potential to detect SMN1 point mutations that are false negative in the NBS programs. By contrast a carrier screening program will not cover homogenously and universally the whole population, the $2 / 0$ carriers, de novo mutations as well as non-biological filiation will contribute with cases that would be detected by universal NBS.

\section{Economic and psychological cost of NBS and parental views}

Mickaël Hiligsmann provided the rationale and background for the economic evaluation of NBS for SMA. Considering the limited healthcare resources available, it is important for decision makers to efficiently allocate scarce healthcare resources [49]. As part of a health technology assessment, economic evaluations provide a framework to identify and compare the costs and effects of potential interventions. These evaluations inform efficient healthcare 
allocation and play an increasing role in pricing and reimbursement decisions and are formally requested in many countries [50].

An economic evaluation can be defined as a comparative analysis between two or more interventions in terms of costs and outcomes [51]. The most common type of economic evaluation is a cost-utility analysis where the outcomes are expressed in quality-adjusted life years (QALYs) that correspond to the number of years in good health and allow capture of the effects of health interventions on both mortality and morbidity. The results of an economic evaluation are typically expressed in terms of an incremental cost-effectiveness ratio defined as the difference in terms of costs between two interventions divided by their difference in effectiveness. An incremental cost-effectiveness ratio represents the additional cost of an intervention per effectiveness unit (e.g., QALY gained) versus the comparator. To qualify an intervention as cost-effective, the incremental cost-effectiveness ratio is compared to a cost-effectiveness threshold representing the decision makers' willingness to pay per effect unit. If the incremental cost-effectiveness ratio falls below the cost-effectiveness threshold, the intervention is considered as cost-effective. Cost-effective thresholds are estimated, for example, at $\$ 100,000$ to $\$ 150,000$ per QALY gained in the USA [52] and are commonly higher for orphan drugs, end of life diseases, and very severe diseases.

Few economic evaluations have been conducted in the field of SMA although there have been some reports about the cost-effectiveness of Spinraza for reimbursement decisions [53], a report of the Institute for Clinical and Economic Review in the USA [54], and one scientific article to assess the cost-effectiveness of Spinraza in Sweden [55]. These studies reported a high incremental cost-effectiveness ratio of an SMA drug (by example estimated at $€ 551,300$ and $€ 311,800$ for Spinraza for the infantile-onset model and later-onset model, respectively) [55] suggesting that, at current prices, SMA drugs are not cost-effective at common thresholds for cost-effectiveness. However, alongside economic considerations, other criteria are important for drug value assessment including the lack of alternative treatment, the societal impact, ethical considerations, and budget impact. The Institute for Clinical and Economic Review in the USA has recognized the high benefits of Spinraza and Zolgensma and the need to consider contextual issues and broader benefits for patients and families in the judgment of overall drug value [54]. A value framework developed specifically for SMA should take into account, in addition to QALY and net costs, factors such as equity, real option value, value of hope, and severity of disease [56].

The one economic evaluation of screening for SMA conducted to date suggested that screening for SMA is not cost-effective [57]. This study was, however, conducted several years ago when drug treatments were not yet available and thus needs to be updated. Given the increasing use of NBS for SMA worldwide and effective treatment options, alongside the importance of economic considerations, investigating the cost-effectiveness of NBS for SMA is needed. As pre-symptomatic treatment of SMA has been shown to be associated with improved treatment benefits compared to post-symptomatic treatment [11,12], we anticipate that an economic evaluation comparing NBS followed by pre-symptomatic treatment could represent a high economic value compared to no screening followed by post-symptomatic treatment. In summary, given the increasing importance of economic evaluations and increasing interest and use of NBS for SMA, it will be important to investigate the cost-effectiveness of NBS for SMA. This analysis will provide relevant information for policy makers.

Philip Young presented data on the views of the general population and SMA community on NBS in the UK. This project was coordinated by Dr. Felicity Boardman [58] (Warwick Medical School) as part of the Imagining Futures Project and was conducted before the availability of innovative medication. This mixed model study involved qualitative (interviews with the SMA community members) and quantitative (survey-based) analyses. Even in absence of disease-modifying treatment, the majority of participants supported NBS (70\% for the SMA community; $84 \%$ for the general population). The most common reasons cited were (1) it would increase support for children and families; (2) it would facilitate enrolment in clinical trials; and (3) it would allow parents to make informed decisions about future pregnancies. Finally, the data highlighted that participants believed the diagnosis of SMA in newborns was important even if the SMA type could not be definitively determined and even in the absence of effective treatment.

Mencia de Lemus pointed out that NBS is a tool that can help provide the SMA community with the best available options to tackle their disease. There is increasing evidence that the earlier SMA patients are treated, the better the prognosis. SMA is a fatal or, at the very least, a highly debilitating disease that if untreated leads to inevitable and constant decline, and all possible efforts to change that road should be pursued. It was emphasized that NBS should not be viewed as a way to limit innovative medication to only presymptomatic patients. Data from NBS should not preclude access to therapy by patients who are symptomatic.

Laetitia Ouillade gave an overview of the situation in France, where Spinraza is now reimbursed for all patients. She underlined the importance of the medical communication during the diagnosis process, which is even more important in the context of a NBS, as the absence of clinical signs can make a diagnosis difficult to accept for parents. She insisted on the standardized follow up of patients with four copies of SMN2 if no treatment is offered. In all cases, the physician should present and explain the different approved and investigational treatments, clearly balancing expectations and unknown long-term effects, and give parents time to make a choice.

Olga Germanenko explained that families with children with SMA now have high expectations about treatment outcomes that sometimes overpass the actual treatment effect. It is therefore very important to communicate the diagnosis 
and treatment options, possible outcomes, and limitations of treatments, as well as the importance of lifelong monitoring. Communication in the NBS screening process has a high impact and should be clear and transparent and reflect the current level of understanding of SMA and the expected effect of potential treatments.

Although NBS for SMA is not yet widely implemented, especially in countries with gaps in access to treatment, it is necessary to gather best practices on different aspects of NBS (e.g., technical, communication, implementation, clinical, legal, advocacy) into guidelines or recommendations that will be useful for countries where NBS is not yet implemented in order to speed up the worldwide process.

Kacper Rucinski stated that SMA patient organizations are supportive of universal NBS for SMA. However, they are also mindful of a range of issues related to early diagnostics and treatment. Experience shows that neuromuscular centers of excellence are usually able to provide a good standard of care but that there is insufficient expertise available in smaller hospitals (e.g., local neonatal and pediatric wards), and these facilities will play an important role in NBS and in decisions on the provision of care to newly diagnosed newborns. Future deliverables of the working group should include the development of universal recommendations to be issued as a Consensus Statement on standards for SMA NBS programs. Such a reference document, containing guidance on the topics ranging from diagnostic methods to timelines, parent communication, clinician training, and performance monitoring, would be of immense help in the design and introduction of SMA pilots and national screening programs worldwide.

Kristin Stephenson provided an overview of how NBS for neuromuscular disorders is moving forward in the USA, with Pompe disease and SMA both added to the US Recommended Uniform Screening Panel by the US Secretary of Health and Human Services and additional disorders such as Duchenne muscular dystrophy in process for nomination to the Recommended Uniform Screening Panel. Implementation of NBS in the USA is determined on a state-by-state basis. An overview of the mechanisms for adding disorders and policy considerations that impact the inclusion of additional disorders to the individual state testing panels was provided. The Muscular Dystrophy Association's recent interest in NBS is in part due to the nationwide network of multidisciplinary medical clinics across the USA that the Muscular Dystrophy Association supports and that serve as the infrastructure to provide expert clinical care and support to babies with SMA and other neuromuscular diseases and their families who are identified and diagnosed through NBS. Ensuring that uniform screening is implemented across all of the USA and that families have access to the care and support available takes the coordinated activities of multiple stakeholders and collaboration across organizations and entities. In the USA, there has been a significant amount of collaboration among clinicians, researchers, state health laboratory personnel, and patient advocacy groups that is allowing NBS for neuromuscular disease to move forward.

\section{Toward a collaborative effort for data-based decision making for treatment}

There is today a growing data set from Spinraza and Zolgensma studies that demonstrate the benefits of early pre-symptomatic treatment in patients with two or with three copies of SMN2 in comparison with post-symptomatic treatments. The question of treating or not treating patients as a function of SMN2 copy number was initially assessed using a Delphi methodology, and the results led to strong consensus for treating patients with two copies, but results were not clear for patients with three or four copies [59]. Although the benefit of treating patients with more than three copies of SMN2 at birth is not established, and the overall feeling of the attendees was that these patients should be treated, the questions of whether or not patients with four copies of SMN2 should be treated at birth is highly debated [60].

It was unanimously agreed that a better standardization of copy number quantification is needed and that the natural history of the disease in patients with three or four copies of SMN2 must be understood before strongly recommending treatment of these patients. This is particularly true in the context of the not-yet-fully-understood short- and long-term safety profiles of the currently used medications. There was a strong agreement that recommendations should be evidencebased.

\section{Workshop key deliverables}

The attendees defined a working plan (WP) divided into seven work packages in order to provide the data for an evidence-based and data-grounded decision making. To take into consideration a strong request issued by the patients and patient advocates, a WP aimed at establishing best practice and training for NBS was added. Attendees volunteered to participate in the different WPs. The attendees agreed that participation of physicians and scientists outside the group of workshop attendees should be encouraged.

It is likely that data issued from these different WPs will not be available for four to five years and during that time patients with a single or with more than three copies will be detected by NBS. For these subjects, the recommendation of the working group is to carefully evaluate the safety of the available treatments and to individually asses the benefit to risk ratios in these patients taking into account familial background and clinical information. If available, the exploratory evaluation of CMAP, pNF-H and/or other potential biomarkers should be taken into account. The group emphasizes that decision making should be a shared process including clinicians and the patient or patient advocates.

WP1: Best practice for SMN2 copy number quantification including the minimal quality standard for DNA samples for reliable assessment (A. Burghes, E. Bertini, F. Boemer, E. Tizzano, F. D. Tiziano). 
WP2: Identification and re-testing with up-to-date methods samples from patients who have reportedly with four copies of SMN2 and presented with a severe phenotype or with type 1 or 2 SMA or who are asymptomatic, and identification of potential SMN2-related or unrelated modifiers (E. Tizzano, E. Bertini, A. Burghes, F. D. Tiziano).

WP3: Identification in the published literature of patient series with two, three, and four copies of SMN2 matching the best practices as defined in WP1, in order to define the major milestones and timelines for different genotypes (E. Tizzano).

WP4: Establish patient milestones (date at first symptoms, date at diagnosis, and date at death) for patients with four copies using best practice as defined by WP1 and locally assessed (L. Servais).

WP5: Assess the prevalence of patients identified with SMN1 loss in the elder general population (A. Burghes, E. Bertini, E. Tizzano, F. D. Tiziano).

WP6: Develop a European Registry of patients identified by NBS (W. Mueller-Felber, E. Bertini, L. Servais).

WP7: Train physicians in best practices for NBS for SMA; the aim of this working plan is to welcome physicians and scientists committed to gathering strong data to support evidence-based recommendations to join different working groups (W. Mueller-Felber, E. Tizzano, M. de Lemus, L. Servais, F. D. Tiziano).

L. Servais will be responsible for overall coordination of the work plan.

\section{Conclusions}

Several studies have demonstrated the benefits of early treatment of patients with SMA and newborn screening for SMA has been initiated in a number of countries. The workshop provided an overview of the current state of the art of different aspects of NBS and SMA including but not limited to scientific, economic, psychological, and ethical perspectives. The attendees did not try to reach a consensus on recommendations for treatment or NBS best practice, which would reflect the opinion only of workshop attendees, but rather defined a working methodology to move forward on critical questions.

\section{SMA Study Group}

SMA Study Group authors:

Tamara Dangouloff, University of Liege, Belgium

Arthur Burghes, Ohio State University, Columbus $\mathrm{OH}$

Enrico Bertini, Bambino Gesù Children's Hospital, Rome, Italy

François Boemer, University of Liege, Belgium

Mickaël Hiligsmann, Maastricht University, Maastricht, The Netherlands

Wolfgang Mueller-Felber, Ludwig-Maximilian University of Munich, Germany
Francesco Danilo Tiziano, Catholic University of Sacred Heart, Rome, Italy

Philip Young, University of Warwick, Coventry, UK

Eduardo F. Tizzano, University Hospital Valle Hebron, Barcelona, Spain

Laurent Servais, MDUK Neuromuscular Center, University of Oxford, UK \& University of Liege, Belgium.

SMA Study Group patient representatives:

Olga Germanenko, Patient Advocacy Group, SMA Moscow, Russia

Mencia de Lemus, Patient Advocacy Group, SMA Spain, Madrid, Spain

Laetitia Ouillade, Patient Advocacy Group, SMA France, Pessac, France

Kasper Rucinski, Patient Advocacy Group, SMA Poland, Poland

Kristin Stephenson, Muscular Dystrophy Association, Washington, D.C., USA

SMA Study Group pharmaceutical company representatives:

Wildon Farwell, Biogen, Boston, MA, USA

Ksenija Gorni, Roche Pharma Research and Early Development, F. Hoffmann -

La Roche Ltd, Basel, Switzerland

Mikael Hjort, PerkinElmer Finland, Turku, Finland

Imran Kausar, Medical Affairs, London, UK and AveXis, (a division of Novartis, Bannockburn, IL, USA)

\section{Acknowledgments}

The organizers warmly acknowledge the staff of ENMC: Alexandra Breukel, Managing Director and Annelies Zittersteijn, Operational Manager. This Workshop was made possible thanks to the financial support of the European Neuromuscular Centre (ENMC) and ENMC main sponsors: Association Française contre les Myopathies (France), Deutsche Gesellschaft für Muskelkranke (Germany), Muscular Dystrophy Campaign (UK), Muskelsvindfonden (Denmark), Prinses Beatrix Spierfonds (The Netherlands), Schweizerische Stiftung für die Erforschung der Muskelkrankheiten (Switzerland), Telethon Foundation (Italy), Spierziekten Nederland (The Netherlands) and Associated members: Finnish Neuromuscular Association (Finland) and Österreichische Muskelforschung (Austria).

The ENMC is also grateful for the support of MDA USA and SMA Europe.

Special thanks are extended to the members of the ENMC Company Forum: Amicus Therapeutics, AveXis, Biogen, CSL Behring, Ionis Pharmaceuticals, PerkinElmer, Roche, Sanofi Genzyme, Sanquin Plasma Products, Santhera Pharmaceuticals, and other partner organizations for their support of the ENMC workshops.

\section{Abbreviations \\ CMAP Compound Muscle Action Potential \\ DBS Dried Blood Spot}


DNA Deoxyribonucleic Acid

FDA Food and Drug Administration

NBS Newborn Screening

PCR Polymerase Chain Reaction

QALY Quality-Adjusted Life Years

qPCR Quantitative Polymerase Chain Reaction

SMA Spinal Muscular Atrophy

SMN Survival of Motor Neuron

\section{References}

[1] Lefebvre S, Burglen L, Reboullet S, Clermont O, Burlet P, Viollet L, et al. Identification and characterization of a spinal muscular atrophy-determining gene. Cell 1995;80(1):155-65.

[2] Alias L, Bernal S, Fuentes-Prior P, Barcelo MJ, Also E, Martinez-Hernandez R, et al. Mutation update of spinal muscular atrophy in Spain: molecular characterization of 745 unrelated patients and identification of four novel mutations in the SMN1 gene. Hum genet. 2009;125(1):29-39.

[3] Dubowitz V. Sixty years of spinal muscular atrophy: a personal odyssey. Spinal muscular atrophy. Sumner CJ, Paushkin S, Ko C-P, editors. Academic Press; 2017. xvii-xxxi.

[4] Klug C, Schreiber-Katz O, Thiele S, Schorling E, Zowe J, Reilich P, et al. Disease burden of spinal muscular atrophy in Germany. Orphanet J Rare Dis. 2016;11(1):58.

[5] Chabanon A, Seferian AM, Daron A, Pereon Y, Cances C, Vuillerot C, et al. Prospective and longitudinal natural history study of patients with type 2 and 3 spinal muscular atrophy: baseline data Nathis-Sma study. PLoS ONE 2018;13(7):e201004.

[6] Calucho M, Bernal S, Alias L, March F, Vencesla A, Rodriguez-Alvarez FJ, et al. Correlation between SMA type and SMN2 copy number revisited: an analysis of 625 unrelated spanish patients and a compilation of 2834 reported cases. Neuromuscul Disord. 2018;28(3):208-15.

[7] Finkel RS, Mercuri E, Darras BT, Connolly AM, Kuntz NL, Kirschner J, et al. Nusinersen versus sham control in infantile-onset spinal muscular atrophy. New Engl J Med. 2017;377(18):1723-32.

[8] Pechmann A, Konig K, Bernert G, Schachtrup K, Schara U, Schorling D, et al. SMArtCARE - a platform to collect real-life outcome data of patients with spinal muscular atrophy. Orphanet J Rare Dis. 2019;14(1):18.

[9] Aragon-Gawinska K, Seferian AM, Daron A, Gargaun E, Vuillerot C, Cances C, et al. Nusinersen in patients older than 7 months with spinal muscular atrophy type 1: a cohort study. Neurology 2018;91(14):e1312-e13e8.

[10] Pane M, Palermo C, Messina S, Sansone VA, Bruno C, Catteruccia M, et al. Nusinersen in type 1 sma infants, children and young adults: preliminary results on motor function. Neuromuscul Disord. 2018;28(7):582-5.

[11] Gidaro T, Servais L. Nusinersen treatment of spinal muscular atrophy: current knowledge and existing gaps. Dev Med Child Neurol. 2019;61(1):19-24.

[12] De Vivo D, Bertini E, Swoboda KJ, et al. Nusinersen initiated in infants during the presymptomatic stage of spinal muscular atrophy: Interim efficacy and safety results from the Phase 2 NURTURE study. Neuromusc Disord 2019;29(11):842-56.

[13] Mendell JR, Al-Zaidy S, Shell R, Arnold WD, Rodino-Klapac LR, Prior TW, et al. Single-dose gene-replacement therapy for spinal muscular atrophy. N Engl Journal Med. 2017;377(18):1713-22.

[14] Dabbous O., Sproule D., Feltner D.E., Ogrinc F.G., Menier M., Droege M., et al. Event-Free survival and motor milestone achievement following AVXS-101 and nusinersen interventions contrasted to natural history for type I spinal muscular atrophy patients. AAN: S25: Child Neurology: Spinal Muscular Atrophy: Treatments and Outcomes. 2019.
[15] Servais L., Baranello G., Day J.W., Deconinck N., Mercuri E., A. K., et al. FIREFISH part 1: survival, ventilation and swallowing ability in infants with type 1 SMA receiving Risdiplam (RG7916). AAN: S25: Child Neurology: Spinal Muscular Atrophy: Treatments and Outcomes. 2019.

[16] Dangouloff T, Servais L. Clinical evidence supporting early treatment in SMA. Ther Clin Risk Manag. 2019;15:1153-61.

[17] FDA. FDA approves innovative gene therapy to treat pediatric patients with spinal muscular atrophy, a rare disease and leading genetic cause of infant mortality: 05/24/2019; 2019 [Available from: https://www.fda.gov/news-events/press-announcements/fda-approvesinnovative-gene-therapy-treat-pediatric-patients-spinal-muscularatrophy-rare-disease.

[18] Wilson JMG, Jungner G. Principles and practice of screening for disease. Public health paper, 34. World Health Organization; 1968.

[19] Serra-Juhe C, Tizzano EF. Perspectives in genetic counseling for spinal muscular atrophy in the new therapeutic era: early pre-symptomatic intervention and test in minors. Eur J Human Genet. 2019;27(12):1774-82.

[20] Chien YH, Chiang SC, Weng WC, Lee NC, Lin CJ, Hsieh WS, et al. Presymptomatic diagnosis of spinal muscular atrophy through newborn screening. J Pediatr. 2017;190 124-9.e1.

[21] Kraszewski JN, Kay DM, Stevens CF, Koval C, Haser B, Ortiz V, et al. Pilot study of population-based newborn screening for spinal muscular atrophy in new york state. Genet Med. 2018;20(6):608-13.

[22] Boemer F, Caberg JH, Dideberg V, Dardenne D, Bours V, Hiligsmann M, et al. Newborn screening for SMA in Southern Belgium. Neuromuscul Disord. 2019;29(5):343-9.

[23] Prior TW, Krainer AR, Hua Y, Swoboda KJ, Snyder PC, Bridgeman SJ, et al. A positive modifier of spinal muscular atrophy in the SMN2 gene. Am J Hum Genet. 2009;85(3):408-13.

[24] Wu X, Wang SH, Sun J, Krainer AR, Hua Y, Prior TW. A-44G transition in SMN2 intron 6 protects patients with spinal muscular atrophy. Hum Mol Genet. 2017;26(14):2768-80.

[25] Ruhno C, McGovern VL, Avenarius MR, Snyder PJ, Prior TW, Nery FC, et al. Complete sequencing of the SMN2 gene in SMA patients detects SMN gene deletion junctions and variants in SMN2 that modify the SMA phenotype. Hum Genet. 2019;138(3):241-56.

[26] Bernal S, Alias L, Barcelo MJ, Also-Rallo E, Martinez-Hernandez R, Gamez J, et al. The c.859G $>\mathrm{C}$ variant in the SMN2 gene is associated with types II and III sma and originates from a common ancestor. J Med Genet. 2010;47(9):640-2.

[27] Schorling DC, Becker J, Pechmann A, Langer T, Wirth B, Kirschner J. Discrepancy in redetermination of SMN2 copy numbers in children with SMA. Neurology 2019;93(6):267-9.

[28] Feldkotter M, Schwarzer V, Wirth R, Wienker TF, Wirth B. Quantitative analyses of SMN1 and SMN2 based on real-time lightCycler PCR: fast and highly reliable carrier testing and prediction of severity of spinal muscular atrophy. Am J Hum Genet. 2002;70(2):358-68.

[29] Yuan A, Rao MV, Veeranna, Nixon RA. Neurofilaments and neurofilament proteins in health and disease. Cold Spring Harb Perspect Biol 2017;9:a018309.

[30] Petzold A. Neurofilament phosphoforms: surrogate markers for axonal injury, degeneration and loss. J Neurol Sci. 2005;233(1-2):183-98.

[31] Mercuri E, Darras BT, Chiriboga CA, Day JW, Campbell C, Connolly AM, et al. Nusinersen versus sham control in later-onset spinal muscular atrophy. N Engl J Med. 2018;378(7):625-35.

[32] Darras BT, Chiriboga CA, Iannaccone ST, Swoboda KJ, Montes J, Mignon L, et al. Nusinersen in later-onset spinal muscular atrophy: long-term results from the phase $1 / 2$ studies. Neurology 2019;92(21):e2492-ee506.

[33] Human N.F.-H. Simple plex assay for the detection of human neurofilament heavy (NF-H) in serum $\mathrm{pEH}$, and cerebrospinal fluid (CSF) [ProteinSimple assay specification sheet]. Available at: https:// www.proteinsimple.com/documents/NF-H_Specification_Sheet_Rev_B. pdf. Accessed 20 May 2019. 
[34] Darras BT, Crawford TO, Finkel RS, Mercuri E, De Vivo DC, Oskoui M, et al. Neurofilament as a potential biomarker for spinal muscular atrophy. Ann Clin Transl Neurol. 2019;6(5):932-44.

[35] Sumner CJ D.B., Muntoni F., Crawford T.O., Finkel R.S., Mercuri E., De Vivo D.C., et al. Association of phosphorylated neurofilament heavy chain (pNF-H) levels with motor function achievement in individuals with spinal muscular atrophy (SMA) treated with nusinersen. Proceedings of the AAN 2019 - Seventy-first Annual Meeting, May 410, 2019, Philadelphia, PA, USA.

[36] Olsson B, Alberg L, Cullen NC, Michael E, Wahlgren L, Kroksmark AK, et al. NFL is a marker of treatment response in children with SMA treated with nusinersen. J Neurol 2019;266:2129-36.

[37] Winter B, Guenther R, Ludolph AC, Hermann A, Otto M, Wurster CD. Neurofilaments and tau in CSF in an infant with SMA type 1 treated with nusinersen. J Neurol Neurosurg Psychiatry 2019;90:1068-9.

[38] Boemer F, Caberg J, Dideberg V, Beckers P, Marie S, Marcelis L, et al. (S)un (M)ay (A)rise on SMA: l'espoir d'une région sans amyotrophie spinale [(S)un (M)ay (A)rise on SMA: the hope of a region without spinal muscular atrophy]. Rev Med Liege 2019;74(9):461-4.

[39] Czibere L, Burggraf S, Fleige T, Gluck B, Keitel LM, Landt O, et al. High-throughput genetic newborn screening for spinal muscular atrophy by rapid nucleic acid extraction from dried blood spots and 384-well qPCR. Eur J Hum Genet. 2019;90:1068-9.

[40] Wurster CD, Ludolph AC. Nusinersen for spinal muscular atrophy. Ther Adv Neurol Disord. 2018;11:1756285618754459.

[41] Mokhtariye A, Hagh-Nazari L, Varasteh A-R, Keyfi F. Diagnostic methods for Lysosomal storage disease. Rep Biochem Mol Biol. 2019;7(2):119-28.

[42] Farrell PM, Rosenstein BJ, White TB, Accurso FJ, Castellani C, Cutting GR, et al. Guidelines for diagnosis of cystic fibrosis in newborns through older adults: cystic fibrosis foundation consensus report. J Pediatr. 2008;153(2):S4-S14.

[43] Tridello G, Castellani C, Meneghelli I, Tamanini A, Assael BM. Early diagnosis from newborn screening maximises survival in severe cystic fibrosis. ERJ Open Res. 2018;4(2):00109-2017.

[44] Amato A, Giordano PC. Screening and genetic diagnosis of hemoglobinopathies in southern and northern europe: two examples. Mediterr J Hematol Infect Dis. 2009;1(1):e2009007.

[45] Abuli A, Boada M, Rodriguez-Santiago B, Coroleu B, Veiga A, Armengol L, et al. NGS-Based assay for the identification of individuals carrying recessive genetic mutations in reproductive medicine. Hum Mutat. 2016:37(6):516-23.

[46] Alias L, Barcelo MJ, Bernal S, Martinez-Hernandez R, Also-Rallo E, Vazquez C, et al. Improving detection and genetic counseling in carriers of spinal muscular atrophy with two copies of the SMN1 gene. Clin Genet. 2014;85(5):470-5.
[47] Luo M, Liu L, Peter I, Zhu J, Scott SA, Zhao G, et al. An Ashkenazi Jewish SMN1 haplotype specific to duplication alleles improves pan-ethnic carrier screening for spinal muscular atrophy. Genet Med. 2014;16(2):149-56.

[48] Alias L, Bernal S, Calucho M, Martinez E, March F, Gallano P, et al. Utility of two SMN1 variants to improve spinal muscular atrophy carrier diagnosis and genetic counselling. Eur J Hum Genet. 2018;26(10):1554-7.

[49] Jonsson E, Banta D. Treatments that fail to prove their worth. interview by Judy Jones. BMJ 1999;319(7220):1293.

[50] Drummond M, Jonsson B, Rutten F. The role of economic evaluation in the pricing and reimbursement of medicines. Health Policy (New York) 1997;40(3):199-215.

[51] Drummond MF, Sculpher MJ, Claxton K, Stoddart GL, Torrance GW. Methods for the economic evaluation of health care programmes. 4th ed Oxford: Oxford: Oxford University Press; 2015.

[52] Neumann PJ, Cohen JT, Weinstein MC. Updating cost-effectiveness-the curious resilience of the $\$ 50,000$-per-QALY threshold. N Engl J Med. 2014;371(9):796-7.

[53] CADTH Common Drug Reviews. Pharmacoeconomic review report: Nusinersen (Spinraza): (Biogen Canada Inc): Indication: Treatment of patients with 5q SMA. Ottawa (ON): Canadian Agency for Drugs and Technologies in Health; 2018.

[54] ICER. Spinraza ${ }^{\circledR}$ and zolgensma $^{\circledR}$ for spinal muscular atrophy: effectiveness and value. 2019. Institute for Clinical and Economic Review, www.icer.org 0403192019 i-iii.

[55] Zuluaga-Sanchez S, Teynor M, Knight C, Thompson R, Lundqvist T, Ekelund M, et al. Cost effectiveness of nusinersen in the treatment of patients with infantile-onset and later-onset spinal muscular atrophy in Sweden. PharmacoEcon. 2019;37(6):845-65.

[56] Garrison LP, Jackson T, Paul D, Kenston M. Value-Based pricing for emerging gene therapies: the economic case for a higher cost-effectiveness threshold. J Manag Care Spec Pharm 2019;27:795-9.

[57] Little SE, Janakiraman V, Kaimal A, Musci T, Ecker J, Caughey AB. The cost-effectiveness of prenatal screening for spinal muscular atrophy. Am J Obstet Gynecol 2010;202(3):253.e1-253.e7.

[58] Boardman FK, Young PJ, Griffiths FE. Population screening for spinal muscular atrophy: a mixed methods study of the views of affected families. Am J Med Genet A 2017:173(2):421-34.

[59] Glascock J, Sampson J, Haidet-Phillips A, Connolly A, Darras B, Day J, et al. Treatment algorithm for infants diagnosed with spinal muscular atrophy through newborn screening. J Neuromuscul Dis 2018;5(2):145-58.

[60] Darras BT, De Vivo DC. Precious SMA natural history data: a benchmark to measure future treatment successes. Neurology 2018;91(8):337-9. 